\section{Berufsverband der Deutschen Urologen e.V.}

Urologe 2012 · 51:1778

DOI 10.1007/s00120-012-3067-3

๑) Springer-Verlag Berlin Heidelberg 2012
Redaktion

W. Bühmann,Wenningstedt/Sylt

\title{
$+\#$ URO-Telegramm
}

\section{Eunuchen-Studie: Hoden verloren, Lebenszeit gewonnen}

Männer sterben im Mittel früher als Frauen. Dieses Manko lässt sich offenbar durch einen kleinen Eingriff beheben: die Kastration. Aus Tierversuchen ist bekannt, dass eine Kastration und der damit verbundene Verlust an männlichen Sexualhormonen das Leben der betroffenen Tiere verglichen mit der Lebensspanne nicht kastrierter männlicher Artgenossen verlängert. Am Menschen ist das bisher wenig erforscht worden. Die Annahme, dass dies wenigstens zum Teil auf einen Mangel an Freiwilligen für einschlägige Studien zurückzuführen ist, dürfte nicht weit neben der Wahrheit liegen.

Koreanische Forscher haben deshalb einen historischen Kniff angewendet und sich in vergangenen Jahrhunderten umgesehen, in denen die Kastration nicht unbedingt freiwillig gewesen war. Hierfür zogen sie die Daten der Yang-Se-Gye-Bo heran, eines zu Beginn des 19. Jahrhunderts niedergeschriebenen Stammbaums von Eunuchen über 13 Generationen [Min KJ et al. Current Biology 2012, 22: R1-2]. Stammbaum und Eunuchen klingt zunächst widersinnig, ist es aber nur halb. Denn in Korea war den Eunuchen die Heirat ebenso erlaubt wie das Adoptieren von Kindern - Mädchen oder kastrierten Knaben, versteht sich. Aus der Genealogie ließen sich die Lebensspannen von 81 Eunuchen errechnen. Im Mittel wurden sie 70 Jahre alt, bei einem Bereich von 29 bis 109 Jahren. Damit verglichen wurden die Lebenszeiten von nicht kastrierten Männern aus drei Familien, die unter ähnlich privilegierten Umständen lebten wie die Eunuchen selbst. Das durchschnittlich erreichte Alter lag hier nur bei rund 56
Jahren, 14 Jahre weniger als bei den hodenlosen Zeitgenossen.

„Die Ergebnisse unserer Studie sprechen für eine Art Tauschgeschäft zwischen Reproduktion und Altern", schreiben die koreanischen Forscher Hiernach würde die Reproduktionsfähigkeit auf Kosten somatischer Reparaturmechanismen aufrechterhalten. Kurz: Testosteron senkt die Lebenserwartung. Ob diese Erkenntnis praktische Folgen haben und die Zahl der Hodenverweigerer steigen wird, bleibt abzuwarten.

Autor: Dr. Robert Bublak Quelle: Uro-News 2012; 16 (11-12): 8

\section{대내배내배}

\section{Jetzt sicher: Zur neuen GOÄ keine Einigung in Sicht}

Nach dem gescheiterten Einigungsversuch im August lecken BÄK und PKV-Verband offenbar weiter ihre Wunden. Ein gemeinsamer Vorschlag zur GOÄ ist nicht in Sicht.In dieser Legislaturperiode wird es keine neue Gebührenordnung für Ärzte (GOÄ) mehr geben. Das hat Dr. Bernhard Rochell, Hauptgeschäftsführer der Bundesärztekammer (BÄK), beim 4 . Bundeskongress Privatmedizin in Köln bestätigt.

Zwischen dem Verband der Privaten Krankenversicherung und der BÄK gebe es auch nach der ergebnislosen Klausurtagung über die Ausgestaltung der neuen GOÄ weiter Gespräche. Es seien noch "vielfältige Beratungsthemen" vorhanden, so Rochell. Ein gemeinsamer Vorschlag von BÄK und PKV-Verband sei nicht in Sicht.

\section{Zur Not ein eigenes GOÄ-Modell} „Wir meinen, dass man der Politik etwas vorlegen sollte, damit es vorangeht. Zur Not wird die Bundesärzte- kammer einen eigenen Weg gehen und ein Modell für die neue GOÄ vorlegen", so Rochell weiter. "Wir müssen das Zwei-Säulen-Modell mit GKV und PKV zukunftsfest machen." Eine „EBM-isierung" der GOÄ werde es mit der Bundesärztekammer nicht geben, betonte Rochell. Bei einer zu starken Annäherung der beiden Gebührenordnungen "stellt sich die Frage nach der Existenzberechtigung der PKV".

\section{Kleiner Einblick in die BÄK-Pläne}

Rochell gab den Kongressbesuchern auch einen kleinen Einblick in die Planungen der BÄK zur GOÄ. Es müsse weiterhin auch eine Einzelleistungsvergütung geben, wenn auch teilweise zusammengefasst in Leistungskomplexen. Die Bedürfnisse der PKV, einen Zeitbezug zu den Leistungen herzustellen, seien nachvollziehbar.
"Auch der Gebührenrahmen muss bestehen bleiben, es ist nur die Frage der Spannbreite bei den Faktoren." Diese werde möglicherweise geringer. „Aber dann muss es auch eine realistische Basisvergütung geben, damit man nicht erst mit 2,3-fachem Faktor auf eine angemessene Vergütung kommt."

„Analogbewertungen machen das Leben mit der guten alten Tante GOÄ zunehmend schwer", beschrieb Rochell den aktuellen Zustand. Mit der neuen GOÄ müsse es das auch weiterhin geben, allerdings weniger als heute, da Innovationen regelmäBig in die GOÄ aufgenommen werden sollen, führte der BÄK-Hauptgeschäftsführer aus.

Autor: Hauke Gerlof Quelle: Ärzte Zeitung online

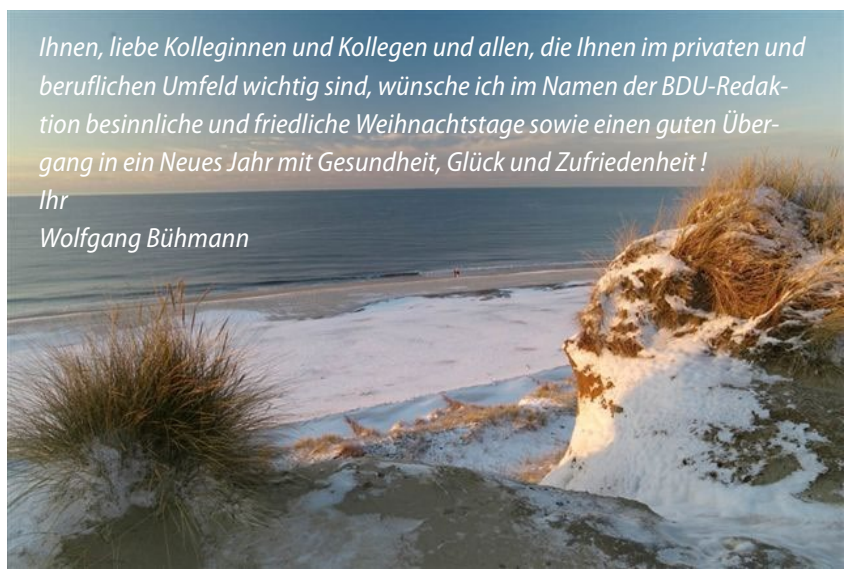

